\title{
Idelalisib and Rituximab in 17p Deletion-Positive Splenic Marginal Zone Lymphoma
}

\author{
Aby Z. Philip, MBBS
}

\section{Abstract}

Splenic marginal zone lymphoma (SMZL) is a rare indolent B-cell malignancy involving the spleen and bone marrow. Various cytogenetic abnormalities with prognostic value have been identified in SMZL. Complexity of karyotype, 14q aberrations, and TP53 deletions have been found to be poor prognostic indicators. We report an unusual case of SMZL with a complex karyotype including 17p deletion, primarily refractory to 2 chemoimmunotherapy regimens, that responded well to treatment with phosphatidylinositol-3-kinase delta (PI3K $\delta$ ) inhibitors idelalisib and rituximab.

J Natl Compr Canc Netw 2018;16(3):230-233 doi: 10.6004/jnccn.2017.7034

Splenic marginal zone lymphoma (SMZL) is a rare lowgrade B-cell lymphoma considered a distinct entity in the WHO classification. ${ }^{1}$ SMZL accounts for approximately $0.6 \%$ of all non-Hodgkin's lymphomas (NHLs), according to the SEER registries, and $<2 \%$ of all lymphoid malignancies. ${ }^{2}$ Patients typically present with an enlarged spleen and involvement of splenic hilar lymph nodes and bone marrow. Extrahilar lymphadenopathy is uncommon.

We report an unusual case of SMZL with diffuse lymphadenopathy and a complex karyotype including $17 \mathrm{p}$ deletion that was refractory to standard chemoimmunotherapy, but responded well to therapy with the phosphatidylinositol-3-kinase delta (PI3Kס) inhibitors idelalisib and rituximab.

\section{Case Presentation}

A previously healthy 68-year-old woman presented with fatigue, night sweats, abdominal fullness, loss of appetite, and low-grade fevers. CT scan of her chest, abdomen, and pelvis revealed diffuse lymphadenopathy involving supraclavicular, axillary, mediastinal, inguinal, retroperitoneal, and mesenteric nodes, and marked splenomega-

From the Hematology \& Oncology Department, Essentia Health Cancer Center, Duluth, Minnesota.

Submitted July 29, 2017; accepted for publication September 11, 2017.

Dr. Philip has disclosed that he has no financial interests, arrangements, ly and ascites. Core needle biopsy of a left axillary node revealed MZL. The neoplastic lymphocytes marked as CD20-positive B cells coexpressed BCL2 and IgD and were kappa immunoglobulin light chain restricted. Test results were negative for CD5, CD10, BCL6, and cyclin D1. Approximately $20 \%$ of the neoplastic B-cell nuclei were $\mathrm{Ki}-67$-positive, indicating a low proliferation rate. Ascitic fluid study showed numerous lymphocytes, with a similar immunophenotypic staining pattern as the lymph node biopsy.

Bone marrow biopsy confirmed SMZL showing light chain restriction and $\mathrm{IgD}$ positivity, involving $90 \%$ of marrow, and decreased trilineage hematopoiesis. Chromosome analysis revealed complex cytogenetics: 45, X, -X, del(2)(p13), add(3)(q12), $\operatorname{add}(3)(\mathrm{q} 12),-17$, $\operatorname{add}(19)(\mathrm{p} 11), \quad+\operatorname{der}(?) \mathrm{t}(? ; 3)(? ; q 11.1)[10] / 46, \quad X X[10]$. Of 20 metaphases, 10 were normal and 10 had a complex karyotype including apparent monosomy 17 (with loss of the TP53 gene region). PET/CT scan ruled out transformation to aggressive histology and showed diffuse FDG-avid adenopathy in cervical, bilateral axillary, mediastinal, retroperitoneal, pelvic sidewall, and inguinal regions, and also throughout the marrow. A maxi-

affiliations, or commercial interests with the manufacturers of any products discussed in this article or their competitors.

Correspondence: Aby Z. Philip, MBBS, Hematology \& Oncology Department, Essential Health Cancer Center, 523 North Third Street, Brainerd, MN 56401. E-mail: aby.philip@essentiahealth.org 
Idelalisib in SMZL With 17p Deletion

mum standardized uptake value of 3.7 was noted in the enlarged spleen.

Chemoimmunotherapy with bendamustine and rituximab was initiated. Paracentesis was performed frequently. Follow-up PET/CT scan after 2 cycles showed disease progression. The patient was seen at a referral center for a second opinion and underwent laparoscopic splenectomy and peritoneal catheter placement. Pathologic examination of the spleen revealed extensive involvement by previously diagnosed SMZL.

Given the progression noted on the PET/CT scan, treatment was changed to rituximab plus cyclophosphamide/doxorubicin/vincristine/prednisone (R-CHOP). After 3 cycles, follow-up CT showed progression of adenopathy, as well as mesenteric caking and interval development of a moderate right pleural effusion. The patient continued to have moderate drainage from the peritoneal catheter and was started on albumin infusions multiple times weekly to correct hypoalbuminemia.

Given her refractory disease and $17 \mathrm{p}$ deletionpositive status, we decided to initiate treatment with idelalisib at the recommended dose of $150 \mathrm{mg}$ twice daily. Follow-up CT scan after 2 cycles showed marked improvement in the extensive adenopathy and resolution of right pleural effusion. Drainage from the peritoneal catheter improved significantly and albumin infusions were discontinued. She tolerated idelalisib well without any significant issues, except for one episode of mild neutropenia that resolved with temporary discontinuation of the drug.

Five months into treatment with single-agent idelalisib, the patient reported onset of B symptoms, such as increased night sweats, as well as increased drainage from her peritoneal catheter. CT scan showed interval increase in diffuse adenopathy involving axillary, anterior, and middle mediastinal; central mesenteric; periaortic; iliac chain; and inguinal regions. Rituximab was added to idelalisib and administered every other week for 4 doses, and every 4 weeks thereafter. Follow-up CT scan after 2 doses of rituximab showed interval response to therapy. B symptoms and ascitic fluid drainage improved, and the peritoneal catheter was subsequently removed.

CT imaging performed 4 months after addition of rituximab to idelalisib showed complete resolution of previously noted diffuse lymphadenopathy and no evidence of progression or new sites of disease. At the time of writing, the patient is continuing on the combination without any adverse effects or complications.

\section{Discussion}

SMZL is considered a low-grade lymphoma with an indolent clinical course. The Italian Lymphoma Intergroup proposed a system of scoring for the assessment of SMZL using 3 laboratory variables (hemoglobin level $<12 \mathrm{~g} / \mathrm{dL}$, elevated serum dehydrogenase level, and albumin level $<3.5 \mathrm{~g} / \mathrm{dL}$ ). ${ }^{3}$ Patients were categorized as low, intermediate, or high risk based on the number of adverse factors present. The 5-year cause-specific overall survival rates for the 3 categories were $88 \%, 73 \%$, and $50 \%$, respectively.

Subsequently, an international retrospective study of 593 patients with SMZL conducted by Montalbán et $\mathrm{al}^{4}$ identified hemoglobin concentration, platelet count, high lactate dehydrogenase (LDH) level, and extrahilar lymphadenopathy (outside the spleen and hepatic hila) as parameters independently associated with lymphoma-specific survival (LSS). In a subsequent study, the same group established clinically acceptable cut points as $9.5 \mathrm{~g} / \mathrm{dL}$ for hemoglobin and $80 \times 10^{9}$ for platelet count. ${ }^{5}$ The LSS for the 3 risk groups were $95 \%$ for the low-risk (defined as having 0 risk factors), $87 \%$ for intermediate-risk ( 1 or 2 risk factors), and $68 \%$ for high-risk groups (3 or 4 risk factors). ${ }^{6}$

Cytogenetic data have been reported in several series of SMZL. Chromosomal aberrations have been detected in approximately $80 \%$ of patients with SMZL. ${ }^{7}$ The most frequent aberrations are gains of $3 \mathrm{q}$ (20\%-30\% of cases) and 12 q (15\%-20\% of cases), and deletion of 7q22-36, mostly at band 7q32 (30\%$40 \%)$. The chromosomes most frequently involved are $1,3,6,7,8,12$, and 14. A multicenter study of $330 \mathrm{pa}$ tients with SMZL evaluating cytogenetic aberrations and their prognostic value demonstrated complexity of the karyotype, 14q aberrations, and TP53 deletions as poor prognostic indicators. ${ }^{8}$ Currently, there are no standardized prognostic factors or any standard treatments based on specific adverse features.

Our patient was classified as high risk due to the presence of 3 risk factors: low hemoglobin level, presence of extrahilar lymphadenopathy, and an elevated LDH level. She also had complex cytogenetics with multiple aberrations, including TP53 deletion, both of which are poor prognostic indicators. 
Management options for SMZL include splenectomy, chemotherapy, rituximab alone, or rituximab with chemotherapy. ${ }^{3}$ Rituximab monotherapy has been shown to be very effective in most patients with SMZL, with overall response rates (ORRs) of $90 \%$ to $100 \%, 9,10$ and has been proposed as a reasonable firstline therapy. ${ }^{11}$

Our patient had symptomatic widespread SMZL and hence was treated with chemoimmunotherapy as first-line therapy. Chemotherapy in combination with rituximab has shown high response rates in SMZL, as seen in the FIL and BRIGHT trials. ${ }^{12,13}$ In the FIL trial, rituximab with cyclophosphamide, nonpegylated liposomal doxorubicin, and prednisone (R-COMP) showed a response rate of $84 \% .{ }^{12}$ In the BRIGHT study, which evaluated efficacy and safety of combination bendamustine and rituximab versus standard R-CHOP/R-CVP (rituximab plus cyclophosphamide/ vincristine/prednisone) regimens in indolent NHL, ORRs for the MZL subgroup was $92 \%$ in the experimental arm compared with $71 \%$ with the standard regimens. ${ }^{13}$

Clinical trials evaluating novel agents specifically in SMZL are few. However, novel agents such as vorinostat, ibrutinib, and idelalisib have been studied in indolent lymphoma, and these trials have included a subset of patients with MZL. Vorinostat demonstrated an ORR of $22 \%$ as a single agent ${ }^{14}$ in the MZL subgroup of relapsed/refractory $(\mathrm{R} / \mathrm{R})$ indolent NHL. Ibrutinib showed an ORR of $25 \%$ in a similar subset of patients with R/R MZL. ${ }^{15}$ Ibrutinib was granted accelerated FDA approval in January 2017 for the treatment of MZL following treatment with at least one prior anti-CD20-based therapy, based on the PCYC-1121 trial. ${ }^{16}$ This multicenter, open-label, single-arm, phase
II trial demonstrated an ORR of 46\% in MZL and 50\% in the SMZL subtype. Idelalisib, a first-in-class oral inhibitor of PI3Kס, showed an ORR of $47 \%$ in the MZL subgroup in a phase II study of R/R indolent NHL. ${ }^{16}$ Venetoclax, a highly sensitive BCL-2 inhibitor, also demonstrated activity in R/R MZL in a phase I tri$\mathrm{al},{ }^{17}$ although the number of patients with MZL in the study was small.

Our patient had SMZL refractory to bendamustine/rituximab and R-CHOP, and therefore we turned to novel agents. Ibrutinib had not been approved yet for MZL. Based on the available clinical trial data and known activity of idelalisib in $17 \mathrm{p}$ deletion-positive tumors, we decided to start her on idelalisib at the standard dose of $150 \mathrm{mg}$ twice daily. Consistent with results from the study by Gopal et al, ${ }^{16}$ our patient's response to idelalisib monotherapy lasted 5 months.

Clinical trials exploring use of novel agents with rituximab have also shown promise in indolent NHL, including MZL. Combinations of idelalisib with rituximab, bendamustine, or both, were found feasible and active in patients with $\mathrm{R} / \mathrm{R}$ indolent NHL in a multicenter phase I trial, with demonstrated ORRs of $75 \%, 88 \%$, and $79 \%$, respectively. ${ }^{18}$ The median progression-free survival (PFS) was 29.7, 32.8, and 37.1 months, respectively. The observed toxicity profile was consistent with experience in previous trials with these drugs in the advanced disease setting. Randomized phase III trials evaluating these combinations have been initiated. Combination idelalisib and rituximab has shown high activity in chronic lymphocytic leukemia, including patients with del(17p)/ TP53 mutations, ${ }^{19,20}$ and is approved for the same indication. A phase II trial with combination vorinostat and rituximab in newly diagnosed and $\mathrm{R} / \mathrm{R}$ indolent

\begin{tabular}{|llcccl|}
\hline Table 1. Novel & Agents in MZL & & & & \\
\hline Agent & Patient Status & Patients, N & MZL & ORR & Duration of Response \\
\hline Vorinostat $^{14}$ & R/R & 35 & 9 & $22 \%$ & Median PFS: 18.8 mo \\
\hline Ibritunib15 $^{15}$ & R/R & 56 & 4 & $25 \%$ & NR \\
\hline Idelalisib $^{16}$ & R/R & 125 & 15 & $47 \%$ & Median PFS: 6.6 mo \\
\hline Venetoclax & R/R & 106 & 3 & $67 \%$ & PFS: 2.3 mo and 23.6 mo \\
\hline $\begin{array}{l}\text { Idelalisib + } \\
\text { rituximab }\end{array}$ & R/R & 79 & 5 & $100 \%$ & Median PFS: 29.7 mo \\
\hline $\begin{array}{l}\text { Vorinostat }+ \\
\text { rituximab }\end{array}$ & First line and R/R & 28 & 2 & $50 \%$ & NR \\
\hline $\begin{array}{l}\text { Lenalidomide } \\
\text { rituximab }\end{array}$ & First line & 103 & 27 & $89 \%$ & Median PFS: 53.8 mo \\
\hline
\end{tabular}

Abbreviations: MZL, marginal zone lymphoma; NR, not reached; ORR, overall response rate; PFS, progression-free survival; R/R, relapsed/refractory. 
Idelalisib in SMZL With 17p Deletion

NHL demonstrated a 50\% ORR in the MZL subgroup with acceptable toxicity, ${ }^{21}$ although there were only 2 patients with MZL. Combination lenalidomide and rituximab demonstrated an ORR of $89 \%$ in untreated MZL, with a median PFS of 53.8 months in a phase II trial. ${ }^{22}$ Results of trials with novel agents and combination treatments in patients with indolent lymphomas, including MZL, are summarized in Table 1.

Based on these data, we decided to add rituximab to idelalisib in our patient after her disease progressed on idelalisib monotherapy. Follow-up imaging after just 2 doses of rituximab showed a marked decrease in her lymphadenopathy, and her B symptoms resolved. Follow-up imaging at 4 months confirmed complete remission. The patient is currently tolerating the regimen well without any adverse effects.

\section{Conclusions}

Our case highlights an area of need in the management of a minority of MZLs and subtypes that har- bor poor prognostic factors, such as the TP53 mutation, and that are primarily refractory to standard chemoimmunotherapy regimens. Clinical trials with novel agents are ongoing in this subgroup of indolent lymphomas. The approval of ibrutinib is one of the first steps in this direction. Other agents, such as vorinostat and idelalisib, show activity as monotherapies and in combination with rituximab, with the combinations showing higher response rates and longer PFS durations. Combination lenalidomide and rituximab has shown significant activity in MZL in the first-line setting.

First-line treatment options for SMZL include rituximab monotherapy, splenectomy, or chemoimmunotherapy, with chemoimmunotherapy preferred in disseminated disease. Novel agents are highly effective, especially in combination with rituximab, and should be considered in the R/R setting. Optimal sequencing of novel agents and combinations must be determined in further studies.

\section{References}

1. Jaffe ES. The 2008 WHO classification of lymphomas: implications for clinical practice and translational research. Hematology Am Soc Hematol Educ Program 2009:523-531.

2. Arcaini L, Rossi D, Paulli M. Splenic marginal zone lymphoma: from genetics to management. Blood 2016;127:2072-2081.

3. Arcaini L, Lazzarino $\mathrm{M}$, Colombo $\mathrm{N}$, et al. Splenic marginal zone lymphoma: a prognostic model for clinical use. Blood 2006;107:46434649.

4. Montalbán C, Abraira V, Arcaini L, et al. Risk stratification for splenic marginal zone lymphoma based on haemoglobin concentration, platelet count, high lactate dehydrogenase level and extrahilar lymphadenopathy: development and validation on 593 cases. Br J Haematol 2012;159:164171.

5. Montalban C, Abraira V, Arcaini L, et al. Simplification of risk stratification for splenic marginal zone lymphoma: a point-based score for practical use. Leuk Lymphoma 2014;55:929-931.

6. Kalpadakis C, Pangalis GA, Angelopoulou MK, et al. Validation of the simplified prognostic score for splenic marginal zone lymphoma of the Splenic Marginal Zone Lymphoma Working Group. Leuk Lymphoma 2012;55:2640-2642.

7. Matutes E, Oscier D, Mantalban C, et al. Splenic marginal zone lymphoma proposals for a revision of diagnostic, staging and therapeutic criteria. Leukemia 2007;22:487-495.

8. Salido M, Baró C, Oscier D, et al. Cytogenetic aberrations and their prognostic value in a series of 330 splenic marginal zone B-cell lymphomas: a multicenter study of the Splenic B-Cell Lymphoma Group. Blood 2020;16:1479-1488.

9. Bennett $\mathrm{M}$, Sharma K, Yegena S, et al. Rituximab monotherapy for splenic marginal zone lymphoma. Haematologica 2005;90:856-858.

10. Kalpadakis C, Pangalis GA, Dimopoulou MN, et al. Rituximab monotherapy is highly effective in splenic marginal zone lymphoma. Hematol Oncol 2007;25:127-131.

11. Dreyling $M$, Thieblemont $C$, Gallamini A, et al. ESMO consensus conferences: guidelines on malignant lymphoma. Part 2: marginal zone lymphoma, mantle cell lymphoma, peripheral T-cell lymphoma. Ann Oncol 2013;24:857-877.

12. Iannitto E, Luminari S, Tripodo C, et al. Rituximab with cyclophosphamide, vincristine, non-pegylated liposomal doxorubicin and prednisone as firstline treatment for splenic marginal zone lymphoma: a Fondazione Italiana Linfomi phase II study. Leuk Lymphoma 2015;56:3281-3287.

13. Flinn IW, van der Jagt R, Kahl BS, et al. Randomized trial of bendamustinerituximab or R-CHOP/R-CVP in first-line treatment of indolent NHL or MCL: the BRIGHT study. Blood 2014;123:2944-2952.

14. Kirschbaum M, Frankel P, Popplewell L, et al. Phase II study of vorinostat for treatment of relapsed or refractory indolent non-Hodgkin's lymphoma and mantle cell lymphoma. J Clin Oncol 2011;29:1198-1203.

15. Advani RH, Buggy JJ, Sharman JP, et al. Bruton tyrosine kinase inhibitor ibrutinib (PCI-32765) has significant activity in patients with relapsed/ refractory B-cell malignancies. J Clin Oncol 2013;31:88-94.

16. Gopal AK, Kahl BS, de Vos S, et al. PI3Kd inhibition by idelalisib in patients with relapsed indolent lymphoma. N Engl J Med 2014;370:10081018 .

17. Davids MS, Roberts AW, Seymour JF, et al. Phase I first-in-human study of venetoclax in patients with relapsed or refractory non-Hodgkin lymphoma. J Clin Oncol 2017;35:826-833.

18. de Vos S, Wagner-Johnston ND, Coutre SE, et al. Combinations of idelalisib with rituximab and/or bendamustine in patients with recurrent indolent non-Hodgkin lymphoma. Blood Adv 2016;1:122-131.

19. Furman RR, Sharman JP, Coutre SE, et al. Idelalisib and rituximab in relapsed chronic lymphocytic leukemia. N Engl J Med 2014;370:997-1007.

20. O'Brien SM, Lamanna N, Kipps TJ, et al. A phase 2 study of idelalisib plus rituximab in treatment-naïve older patients with chronic lymphocytic leukemia. Blood 2015;126:2686-2694.

21. Chen R, Frankel P, Popplewell L, et al. A phase II study of vorinostat and rituximab for treatment of newly diagnosed and relapsed/refractory indolent non-Hodgkin lymphoma. Haematologica 2015;100:357-362.

22. Fowler NH, Davis RE, Rawal S, et al. Safety and activity of lenalidomide and rituximab in untreated indolent lymphoma: an open-label, phase 2 trial. Lancet Oncol 2014;15:1311-1318. 